\title{
Stability, Bifurcation, and Quenching Chaos of a Vehicle Suspension System
}

\author{
Chun-Cheng Chen ${ }^{1}$ and Shun-Chang Chang $\mathbb{D D}^{2}$ \\ ${ }^{1}$ Graduate School of Technological and Vocational Education, National Yunlin University of Science and Technology, \\ Yunlin 64002, Taiwan \\ ${ }^{2}$ Department of Mechanical and Automation Engineering, Da-Yeh University, Changhua 51591, Taiwan
}

Correspondence should be addressed to Shun-Chang Chang; changsc@mail.dyu.edu.tw

Received 16 October 2017; Revised 28 January 2018; Accepted 20 February 2018; Published 27 March 2018

Academic Editor: David F. Llorca

Copyright (c) 2018 Chun-Cheng Chen and Shun-Chang Chang. This is an open access article distributed under the Creative Commons Attribution License, which permits unrestricted use, distribution, and reproduction in any medium, provided the original work is properly cited.

\begin{abstract}
This study investigated the dynamics and control of a nonlinear suspension system using a quarter-car model that is forced by the road profile. Bifurcation analysis used to characterize nonlinear dynamic behavior revealed codimension-two bifurcation and homoclinic orbits. The nonlinear dynamics were determined using bifurcation diagrams, phase portraits, Poincaré maps, frequency spectra, and Lyapunov exponents. The Lyapunov exponent was used to identify the onset of chaotic motion. Finally, state feedback control was used to prevent chaotic motion. The effectiveness of the proposed control method was determined via numerical simulations.
\end{abstract}

\section{Introduction}

The suspension system of vehicles is meant to isolate the vehicle body and passengers from oscillations imposed by road irregularities and maintain continuous contact between the tires and surface of the road. Suspension systems provide a smoother ride and help the operator to maintain control of the vehicle over rough terrain or during sudden stops [1]. Hysteresis is an important aspect of suspension design, and the development of smart materials for vehicle suspension systems with hysteretic damping has been the subject of considerable studies [2-5]. The fact that the damping force in a vehicle's suspension system is hysteretic means that road excitation can be modeled as a multifrequency harmonic function. Therefore, automotive suspension systems may be considered a nonlinear hysteretic system with multifrequency excitation.

Nonlinear phenomena of codimension bifurcations have been studied extensively, particularly in engineering systems [6-8]. Tseng and Tung [9] examined the bifurcation behavior of a nonlinear structure with a magnetic actuator. Yagasaki [10] investigated nonlinear dynamics and the bifurcation behavior of a pendulum with feedback control. Tian et al.
[11] studied the complex nonlinear dynamics of smooth, discontinuous oscillators. Numerous methods have been proposed for nonlinear control. Yin et al. [12] applied an approximated adaptive fuzzy control method to a nonlinear strict-feedback system with unmodeled dynamics. And, Yin et al. [13] developed a fault-tolerant controller based on the adaptive neural network backstepping technique for timevarying delay systems with unmodeled dynamics.

The fact that hysteresis is not a smooth process renders the characteristics of a suspension system with hysteretic damping inherently nonlinear. This can greatly complicate the design of a controller. Accurately predicting and/or controlling the performance of a system require that the effects of nonlinear phenomena be taken into account. The problem of rough road profiles and unwanted vehicle vibrations produced by kinematic excitation make it necessary to form accurate predictions of dynamic behavior over a range of operating conditions in the design of suspension systems. Bifurcation and chaos are crucial concepts in the study of stability in nonlinear systems, and numerous researchers have addressed issues pertaining to nonlinear dynamics in suspension systems [14-17] as well as chaotic responses in nonlinear air suspension systems $[18,19]$. However, the 
issue of codimension bifurcations has largely been overlooked.

In this study, we examined a suspension system presenting codimension-two bifurcation and homoclinic orbits, both of which determine the nature of the dynamic behavior and the existence of strange attractors. We sought to calculate the strange attractor using numerical simulation in which external forces from a road profile are added to the system as a parameter with a specific range of values. We employed bifurcation diagrams, phase portraits, Poincaré maps, frequency spectra, and Lyapunov exponents to explain periodic and chaotic motions in a vehicle suspension system that exerts hysteretic nonlinear damping forces. Algorithms that computes Lyapunov exponents for smooth dynamic systems [20] were used to determine whether the system exhibits chaotic motion.

Chaotic behavior is normally considered undesirable due to the restrictions it imposes on the operating range of electri$\mathrm{cal}$ and mechanical devices. Many engineering problems can only be resolved by driving a chaotic attractor in a periodic orbit. Numerous methods have been proposed for the control of chaos [21-23], including suspension systems [15, 24, 25]. In this study, we adopted the simple control method proposed by Cai et al. [26], in which state feedback is used to convert chaotic motion into stable motion. Numerical simulations demonstrate the feasibility of the proposed approach.

This paper is organized as follows. Section 2 describes the proposed vehicle suspension system using a quartercar model under kinematic excitation by a road surface profile. Section 3 outlines the local bifurcation analysis used to identify codimension-two bifurcation and homoclinic orbits. Section 4 elucidates the complex dynamic behavior in the suspension system using numerical analysis, such as a bifurcation diagrams, phase portraits, Poincaré maps, and frequency spectra. Section 5 presents the Lyapunov exponent used to determine whether the system exhibits chaotic motion. A state feedback control technique for controlling chaos in the suspension system is presented in Section 6. Conclusions are drawn in Section 7.

\section{Formulation of Problem}

Figure 1 presents quarter-car model with a single degree-offreedom and hysteretic nonlinear damping. The equation of motion for the system was formulated by Li et al. [14]:

$$
m \ddot{x}+k_{1}\left(x-x_{0}\right)+F_{h}=0,
$$

where $F_{h}$ is an additional nonlinear hysteretic suspension damping and stiffness force, which is dependent on the relative displacement and velocity:

$$
\begin{aligned}
& F_{h}=k_{2}\left(x-x_{0}\right)^{3}+C_{1}\left(\dot{x}-\dot{x}_{0}\right)+C_{2}\left(\dot{x}-\dot{x}_{0}\right)^{3}, \\
& x_{0}=\widetilde{a} \sin (\Omega t) .
\end{aligned}
$$

In defining the relative vertical displacement as $y=x-x_{0}$, (1) can be written as follows:

$$
\ddot{y}+\omega^{2} y+B_{1} y^{3}+B_{2} \dot{y}+B_{3} \dot{y}^{3}=A \sin (\Omega t),
$$

\begin{tabular}{|c|c|c|c|}
\hline Notation & Value & Unit & Description \\
\hline$m$ & 240 & $\mathrm{~kg}$ & The body mass \\
\hline$k_{1}$ & $16 \times 10^{4}$ & $\mathrm{~N} / \mathrm{m}$ & The suspension stiffness coefficient \\
\hline$k_{2}$ & $30 \times 10^{4}$ & $\mathrm{~N} / \mathrm{m}^{3}$ & $\begin{array}{c}\text { The nonlinear hysteretic suspension } \\
\text { stiffness coefficient }\end{array}$ \\
\hline$C_{1}$ & 250 & $\mathrm{Ns} / \mathrm{m}$ & The linear damping coefficient \\
\hline$C_{2}$ & -25 & $\mathrm{Ns}^{3} / \mathrm{m}^{3}$ & $\begin{array}{c}\text { The nonlinear hysteretic suspension } \\
\text { viscous damping coefficient }\end{array}$ \\
\hline$\tilde{a}$ & 0.05 & $\mathrm{~m}$ & The amplitude of road excitation \\
\hline$x_{0}$ & & $\mathrm{~m}$ & The road excitation \\
\hline$x$ & & $\mathrm{~m}$ & The body's vertical displacement \\
\hline$\Omega$ & & $\mathrm{rad} / \mathrm{s}$ & The frequency of road excitation \\
\hline
\end{tabular}

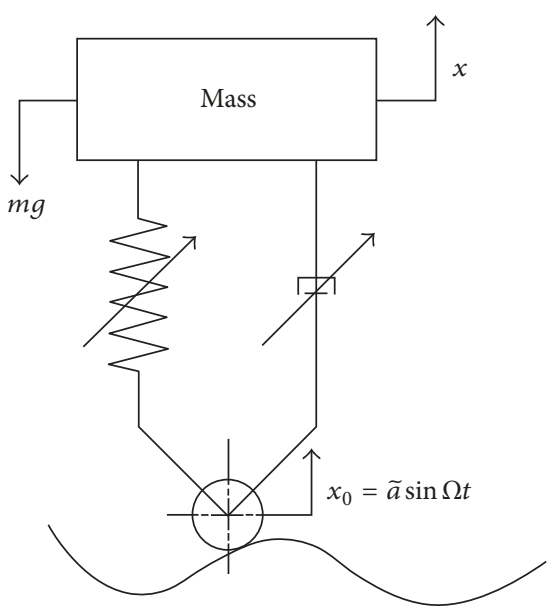

FIGURE 1: Schematic diagram of the quarter-car model.

TABLE 1: Nomenclature and parameters.

where $\omega^{2}=k_{1} / m, B_{1}=k_{2} / m, B_{2}=C_{1} / m, B_{3}=C_{2} / m$, $A=\widetilde{a} \Omega^{2}$.

The states are chosen such that $y_{1}=y, y_{2}=\dot{y}$. Thus, the state-space model for the suspension system can be written as

$$
\begin{aligned}
& \dot{y}_{1}=y_{2}, \\
& \dot{y}_{2}=-\omega^{2} y_{1}-B_{1} y_{1}{ }^{3}-B_{2} y_{2}-B_{3} y_{2}{ }^{3} .
\end{aligned}
$$

The amplitude of the road profile excitation is $\widetilde{a}=$ 0 . Table 1 lists the values of the parameters in the above equations, which were obtained using the methods outlined by Li et al. [14].

\section{Local Codimension-Two Bifurcation Analysis}

Our main objective in this analysis is to observe the qualitative behavior of the system and how it changes as system parameters are varied. Changes occurring near the equilibrium point of the system are referred to as local bifurcation. The codimension of bifurcation refers to the minimum number of parameters that must be varied in order to observe a specific type of bifurcation [8]. 
Nonlinear analysis must be based on linear analysis. Bifurcation analysis is based on the Jacobian matrix in (4a) and $(4 \mathrm{~b})$. When $\left(k_{1}, c_{1}\right)=(0,0)$ and the Jacobian matrix has double-zero eigenvalues of the form of $\left[\begin{array}{ll}0 & 1 \\ 0 & 0\end{array}\right]$, we have the most degenerate situation (eigenvalues with zero real part), that is, a double-zero codimension-two bifurcation problem. Bifurcation equations can be written as follows:

$$
\begin{aligned}
& \dot{y}_{1}=y_{2}, \\
& \dot{y}_{2}=-B_{1} y_{1}^{3}+\text { higher order terms. }
\end{aligned}
$$

Bifurcation equations (5a) and (5b) take the normal form because they are the simplest forms of the equations of motion [8, 27-30].

The blow-up technique is used to check whether the cubic terms in (5a) and (5b) determine local behavior. The blowup technique involves a singular change to the coordinates that expands degenerate fixed points into circles containing a finite number of fixed points. If these fixed points are hyperbolic following the first change in the coordinates, then the local flow near the circle (and consequently flow near the original fixed point) remains stable with respect to higherorder terms. If these fixed points are all degenerate, then the coordinates must be changed again. If all of the fixed points become hyperbolic within a finite number of coordinate changes, then a system with only lower-order terms will exhibit the same qualitative behavior as the overall system [8]. The steps used in the blow-up method are as follows:

First Blow-Up

$$
\begin{aligned}
& y_{1}=r \cos \theta, \\
& y_{2}=r \sin \theta .
\end{aligned}
$$

Equations (5a), (5b) and (6a), (6b) become

$$
\begin{aligned}
& \dot{r}=r \sin \theta\left(1-B_{1} r^{2} \cos ^{2} \theta\right)+\text { h.o.t., } \\
& \dot{\theta}=-\sin ^{2} \theta-B_{1} r^{2} \cos ^{4} \theta+\text { h.o.t. }
\end{aligned}
$$

Stability analysis reveals that the system has two degenerate equilibrium points $(0,0)$ and $(0, \pi)$, which means that the blow-up procedure must be repeated. In the case of $(0,0)$, the power series in normal form is

$$
\begin{aligned}
& \dot{r}=r \theta+\text { h.o.t., } \\
& \dot{\theta}=-\theta^{2}-B_{1} r^{2}+\text { h.o.t.. }
\end{aligned}
$$

Second Blow-Up. Let

$$
\begin{aligned}
& r=\rho \sin \phi, \\
& \theta=\rho \cos \phi,
\end{aligned}
$$

so that ( $8 \mathrm{a}),(8 \mathrm{~b})$ and $(9 \mathrm{a}),(9 \mathrm{~b})$ become

$$
\begin{aligned}
& \dot{\rho}=-\rho^{2} \cos \phi\left(B_{1} \sin ^{2} \phi+\cos ^{2} \phi\right)+\text { h.o.t. } \\
& \dot{\phi}=\rho \sin \phi\left(2 \cos ^{2} \phi-B_{1} \sin ^{2} \phi\right)+\text { h.o.t.. }
\end{aligned}
$$

After dividing by the unaffected common component, $\rho$, (10a) and (10b) have two hyperbolic fixed points, $(0,0)$ and $(0, \pi)$. Thus, no further blow-up is required.

For the other equilibrium point, $(0, \pi)$, the power series in the normal form is the same as the power series used for (8a) and (8b), such that the same results are obtained. The degenerate field contains cubic terms of the lowest order and unfolding is performed using the two-parameter family, as follows:

$$
\begin{aligned}
& \dot{y}_{1}=y_{2}, \\
& \dot{y}_{2}=K_{1} y_{1}+K_{2} y_{2}-B_{1} y_{1}^{3},
\end{aligned}
$$

where $K_{1}=-k_{1} / m, K_{2}=-c_{1} / m$. The higher-order terms in (11a) and (11b) are disregarded when applied as an alternative for (4a) and (4b).

The term "bifurcation set" refers to a set in parameter space on which the system bifurcates. It is obtained from unfolded equations in the normal form.

There are three equilibrium points in this case:

1. $(0,0)$ for all $K_{1}, K_{2}$;

2. $\left( \pm \sqrt{K_{1} / B_{1}}\right)$ for $K_{1}>0$.

By checking the stability of these equilibrium points, we obtain the following:

(1) For $K_{1}=0$, a subcritical pitchfork bifurcation occurs if $K_{2}>0$ and a supercritical pitchfork bifurcation occurs if $K_{2}<0$.

(2) For $K_{1}>0$ and $K_{2}=0$, a rescaling technique and Melnikov's perturbation method are used to obtain the complete bifurcation set associated with homoclinic orbits in the unfolding bifurcation equations (11a) and (11b) [8].

Let $y_{1}=\epsilon u, y_{2}=\epsilon^{2} v, K_{1}=\epsilon^{2} v_{1}, K_{2}=\epsilon^{3} v_{2}, t \rightarrow \epsilon t$.

Thus, after rescaling, (11a) and (11b) are rewritten as

$$
\begin{aligned}
& \dot{u}=v, \\
& \dot{v}=v_{1} u+\epsilon^{2} v v_{2}-B_{1} u^{3} .
\end{aligned}
$$

Melnikov's method is then used to characterize the relationship between $K_{1}$ and $K_{2}$.

After a series of operations, the following expression is obtained:

$$
\begin{aligned}
& K_{2}=0, \\
& K_{1}=B_{1} \epsilon^{3} .
\end{aligned}
$$

Equations (13a) and (13b) yield the homoclinic saddle connection, referred to as homoclinic bifurcation.

(3) For $K_{1}<0$ and $K_{2}=0$, the Jacobian matrix in (11a) and (11b) is written as

$$
\mathrm{DF}(0,0)=\left[\begin{array}{cc}
0 & 1 \\
K_{1} & 0
\end{array}\right]
$$




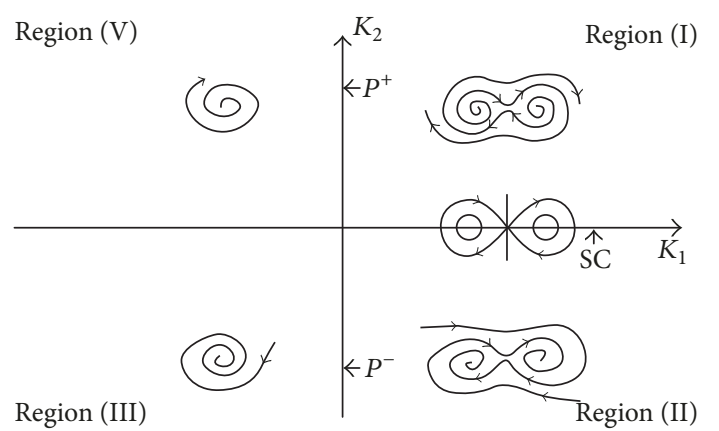

FIGURE 2: The bifurcation sets that are associated with typical phase portraits in each region, where $P^{+}$represents a subcritical pitchfork, $P^{-}$represents a supercritical pitchfork, and SC represents a homoclinic bifurcation.

The eigenvalues for $\operatorname{DF}(0,0)$ are

$$
\lambda= \pm \sqrt{K_{1}} i .
$$

For the following change in coordinates,

$$
\left[\begin{array}{l}
y_{1} \\
y_{2}
\end{array}\right]=\left[\begin{array}{cc}
0 & 0 \\
-\sqrt{K_{1}} & 0
\end{array}\right]\left[\begin{array}{l}
U \\
V
\end{array}\right] .
$$

Equations (12a) and (12b) become

$$
\left[\begin{array}{c}
\dot{U} \\
\dot{V}
\end{array}\right]=\left[\begin{array}{cc}
K_{2} & -\sqrt{K_{1}} \\
\sqrt{K_{1}} & 0
\end{array}\right]\left[\begin{array}{l}
U \\
V
\end{array}\right]+\left[\begin{array}{c}
0 \\
\frac{B_{1} V^{3}}{\sqrt{K_{1}}}
\end{array}\right] .
$$

Let $g(U, V)=B_{1} V^{3} / \sqrt{K_{1}}$ and $f(U, V)=0$.

Clearly, it can be seen that

$$
\begin{aligned}
f_{v v v} & =0, \\
f_{v u u} & =0 \\
g_{v v u} & =0, \\
g_{u u u} & =0 \\
f_{u u} & =0, \\
f_{u v} & =0, \\
f_{v v} & =0 \\
g_{u u} & =0, \\
g_{u v} & =0, \\
g_{v v} & =0 .
\end{aligned}
$$

According to the Hopf bifurcation theorem [8], coefficient a must not equal zero. In this case, the coefficient a equals zero; therefore, there is no Hopf bifurcation.

Numerical simulations using (4a) and (4b) were performed to check the validity of this bifurcation analysis. The parameter space was divided into several regions using bifurcation sets, as shown in Figure 2. The commercial

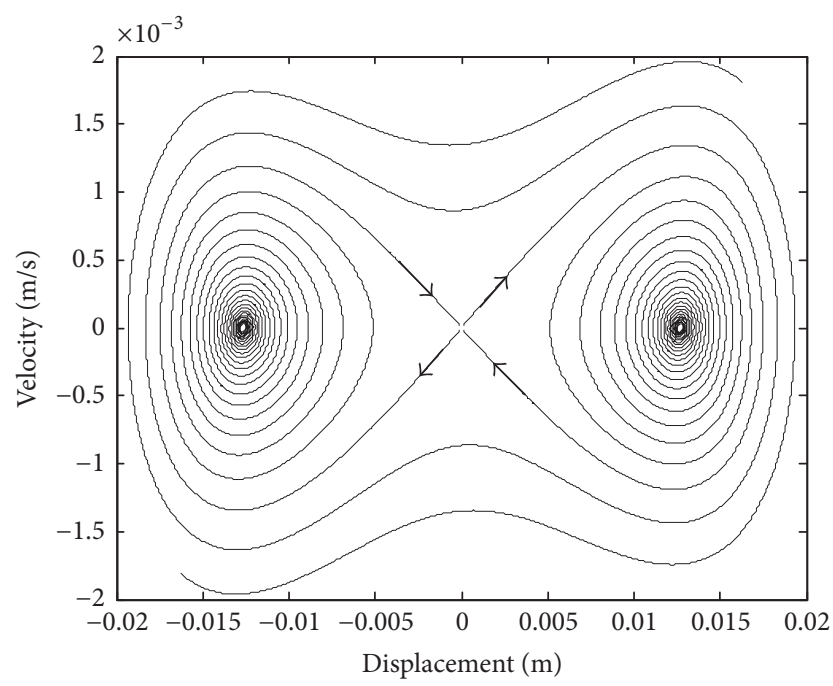

FIGURE 3: Numerical simulation for region (I), for $\left(K_{1}, K_{2}\right)=(0.02$, $0.01)$.

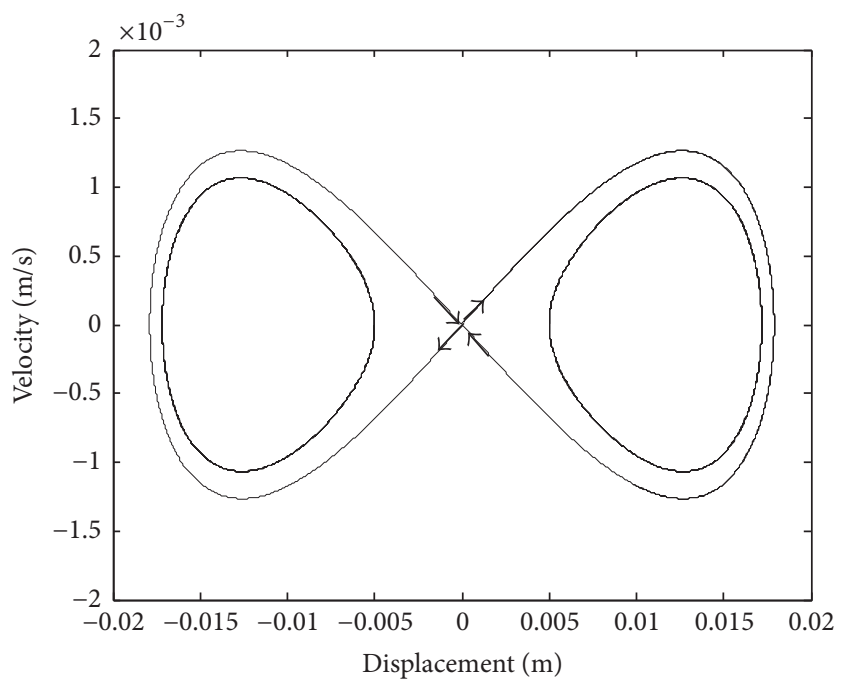

FIgURE 4: Numerical simulation for SC, for $\left(K_{1}, K_{2}\right)=(0.02,0.0)$.

package DIVPRK of IMSL in the FORTRAN subroutines for mathematics applications was used to solve these ordinary differential equations [31]. The resulting numerical simulations are presented in Figures 3-7. The results in each parametric region are discussed below.

Region (I) includes sources and a saddle. If the initial conditions are in the neighborhood of the two sources, then the mass is distant from the equilibrium points (Figure 3 ). At the boundary between regions (I) and (II) (i.e., in the bifurcation set SC $\left.\left(K_{2}=0, K_{1}>0\right)\right)$, the system is an integrable system with homoclinic orbits (Figure 4). Therefore, if the damping term is disregarded, then the system is conservative. In region (II), the system is not integrable with two sinks and one saddle. If the initial conditions are in the neighborhood of the saddle, then the mass returns to the two sinks, which are stable points, as time approaches infinity (Figure 5). At the boundary between regions (II) and 


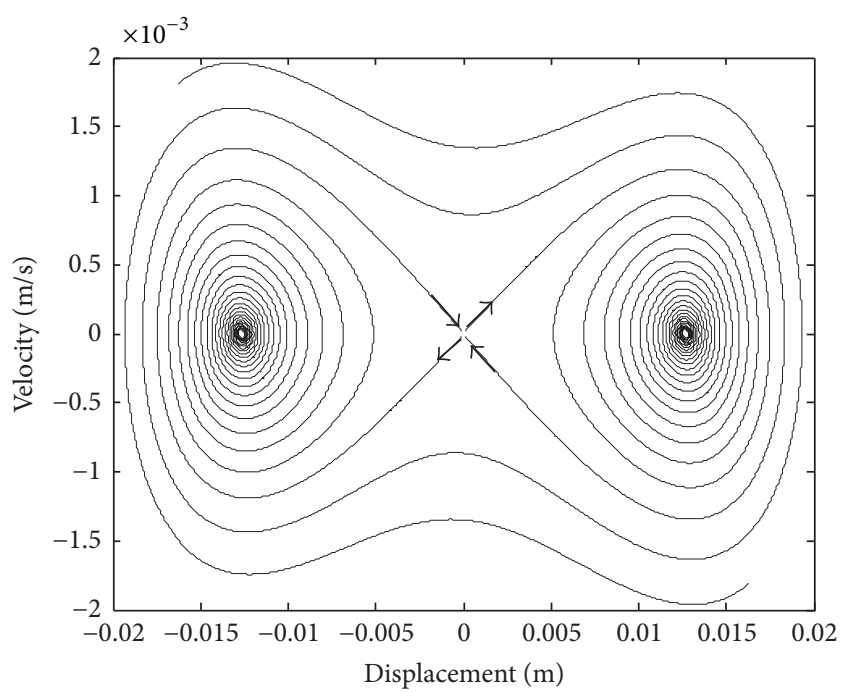

FIGURE 5: Numerical simulation for region (II), for $\left(K_{1}, K_{2}\right)=(0.02$, $-0.01)$.

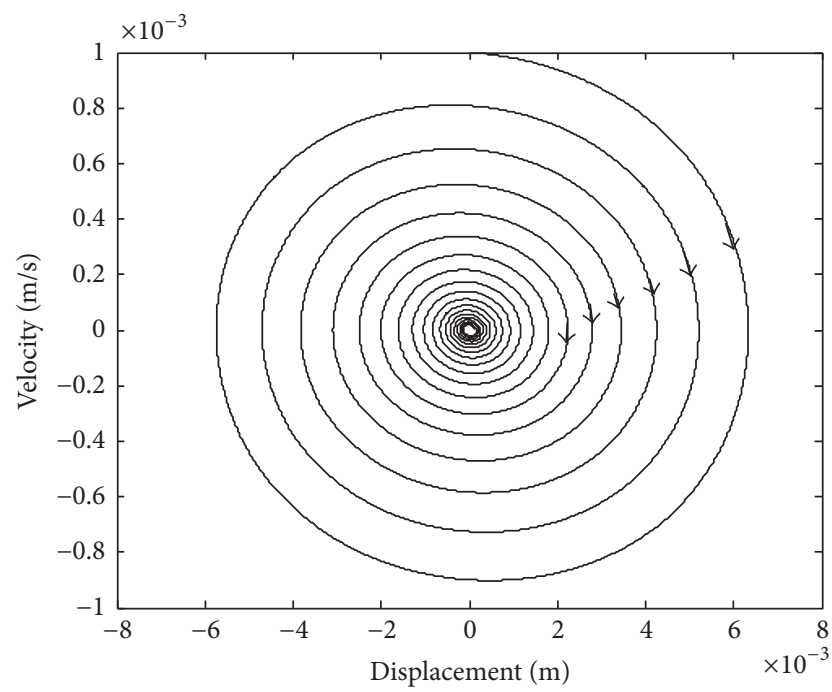

FIgURE 6: Numerical simulation for region (III), for $\left(K_{1}, K_{2}\right)=$ $(-0.02,-0.01)$.

(III) (i.e., in bifurcation set $P^{-}$), the two sinks move closer to the saddle as the parameters approach bifurcation set, $P^{-}$, and the stability region shrinks to zero. In region (III), the two sinks disappear and the saddle $(0,0)$ becomes a sink. This is a supercritical pitchfork bifurcation with an attracting sink region (III) (Figure 6). From regions (III) to (V), the eigenvalues for the Jacobian matrix cross the imaginary axis; however, there is no Hopf bifurcation. This means that the dynamic behavior is linear, which is a similar situation to that when a stable equilibrium point becomes an unstable equilibrium point (Figure 7). Between region (V) and region (I), boundary $P^{+}$must be crossed. This is called a subcritical pitchfork bifurcation.

In terms of the equilibria and Figure $2, K_{1}$ determines the number of equilibrium points and $K_{2}$ determines the

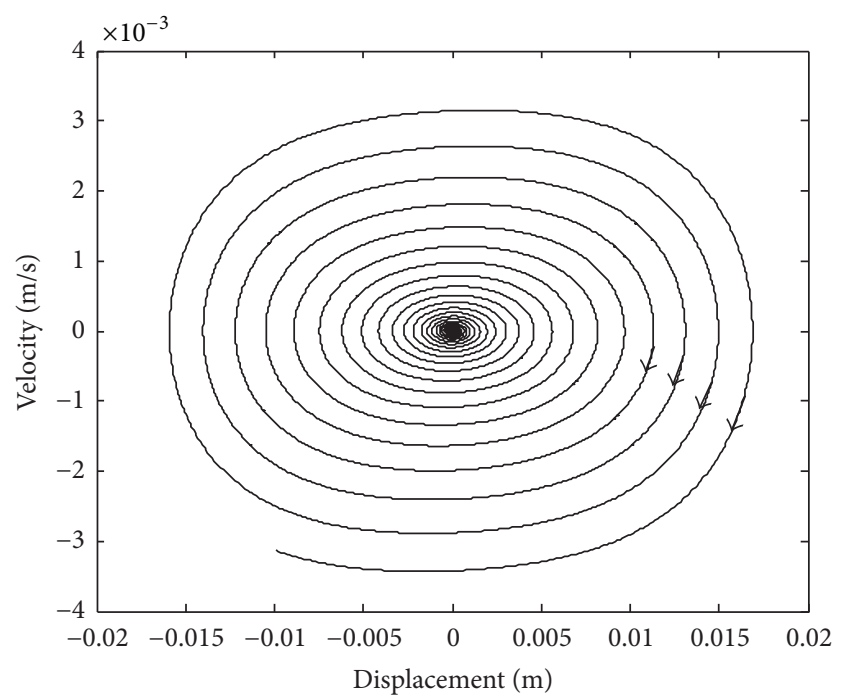

FIgURE 7: Numerical simulation for region $(\mathrm{V})$, for $\left(K_{1}, K_{2}\right)=$ $(-0.02,0.01)$.

local behavior (e.g., stability or instability), and whether there are homoclinic orbits. Clearly, only a positive damping coefficient results in a stable system.

\section{Analytical Tools for Observing Nonlinear Dynamics in a Vehicle Suspension System}

In (3), the amplitude of the road excitation, $\tilde{a}$, equals $0.05 \mathrm{~m}$. We performed a series of numerical simulations using (3) to determine the characteristics of this system. Dynamic behaviors can be more clearly observed over a range of parameter values in bifurcation diagrams, which are widely used to describe transitions from periodic motion to chaotic motion in dynamic systems. The resulting bifurcation diagram is presented in Figure 8. This figure clearly shows chaotic motion in region $\mathbf{A}$. Region $\mathbf{C}$ presents period-1 motion and there is a stable equilibrium point. In this region, each response was characterized using a phase portrait, a Poincaré map (velocity versus phase angle), and a frequency spectrum. Figure 9 shows that the equilibrium point of the system is stable if parameter, $\Omega$, exists in region $\mathbf{C}$. It is wellknown that road excitation covers a wide range frequencies, and higher vehicle velocities increase excitation from the road surface. The range of human sensitivity to frequency is from 4 to $8 \mathrm{~Hz}$ (about $25.12 \mathrm{rad} / \mathrm{s}$ to $50.24 \mathrm{rad} / \mathrm{s}$ ); therefore, we limited this investigation to the case of $\Omega=40.0 \mathrm{rad} / \mathrm{s}$. Figure 10 shows the periodic motion in the system in cases where the parameter exists in region $\mathrm{C}$ (i.e., $\Omega=8.2 \mathrm{rad} / \mathrm{s}$ and $\Omega=40 \mathrm{rad} / \mathrm{s}$ ). The amplitude of road excitation is defined as $0.05 \mathrm{~m}$, while the response of the system (even in periodic motion) has an amplitude of near $0.35 \mathrm{~m}$ (Figure 9). Clearly, this response is not acceptable. When parameter $\Omega$ exists in region $\mathbf{B}$, quasiperiodic motion occurs in this region. As shown in Figures 11-13, the quasiperiodic trajectory is restricted to an annular-like region of the state space and differs considerably from periodic trajectories. When 


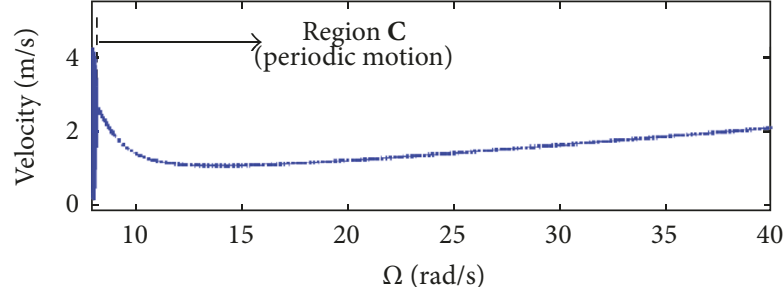

(a)

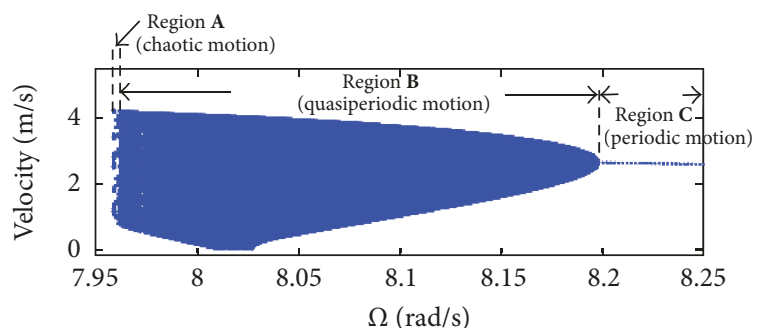

(b)

FIGURE 8: Bifurcation diagram for the system when the amplitude of the road excitation, $\widetilde{a}$, is $0.05 \mathrm{~m}:$ (a) $\Omega=7.96 \mathrm{rad} / \mathrm{s}$ to $40 \mathrm{rad} / \mathrm{s}$, (b) $\Omega=$ $7.96 \mathrm{rad} / \mathrm{s}$ to $8.25 \mathrm{rad} / \mathrm{s}$.

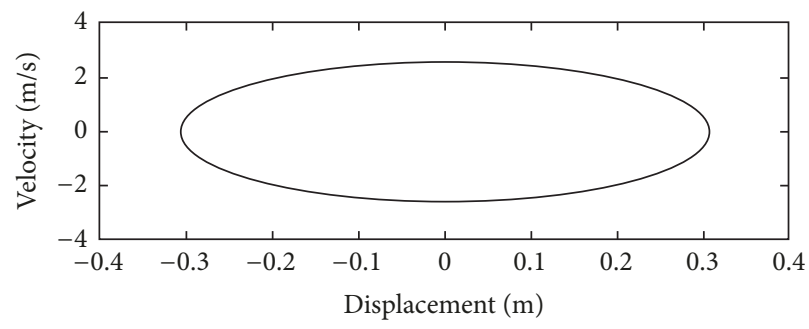

(a)

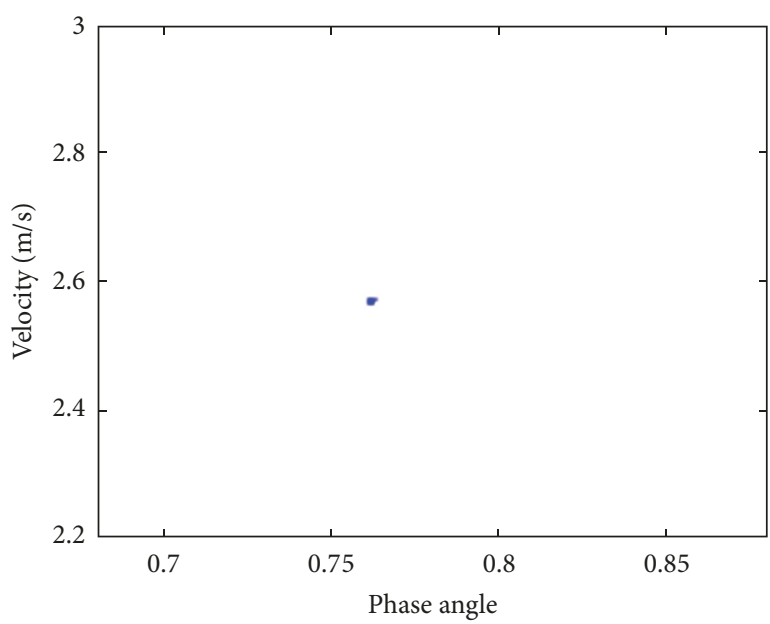

(b)

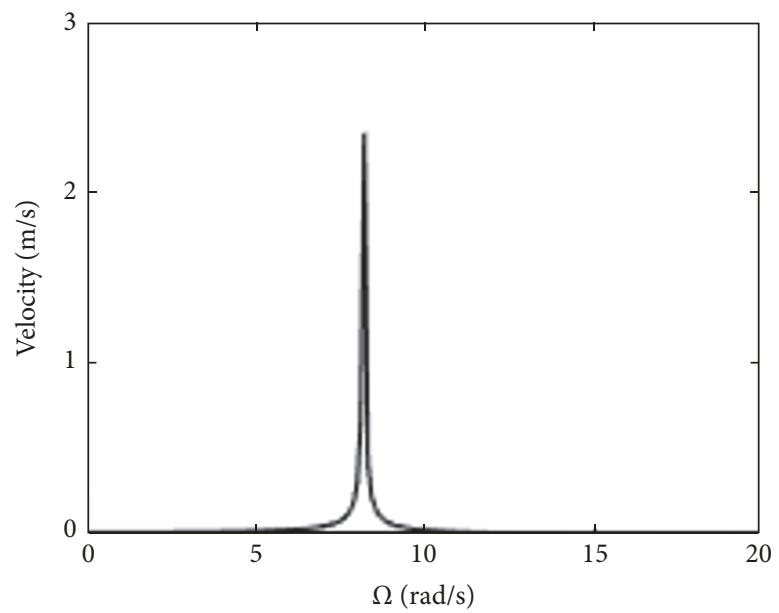

(c)

FIGURE 9: The period-one orbit at $\Omega=8.2 \mathrm{rad} / \mathrm{s}$ : (a) the phase portrait, (b) the Poincaré map, and (c) the frequency spectrum.

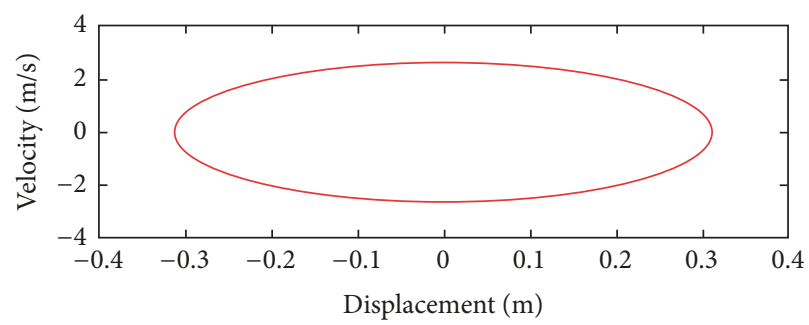

(a)

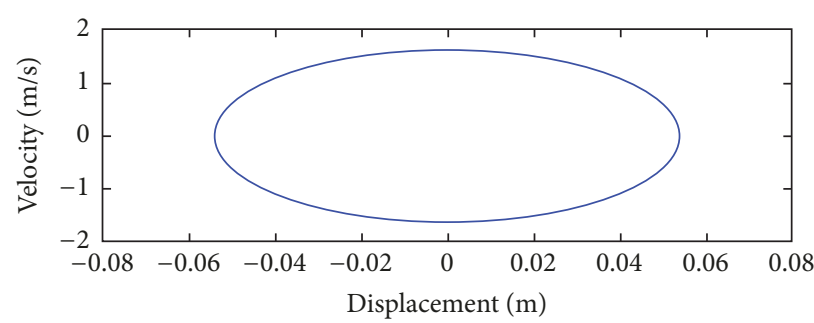

(b)

FIGURE 10: Phase portrait: (a) $\Omega=8.2 \mathrm{rad} / \mathrm{s}$, (b) $\Omega=40 \mathrm{rad} / \mathrm{s}$. 


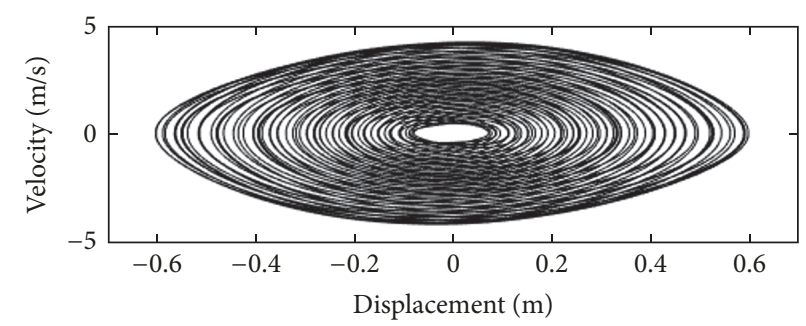

(a)

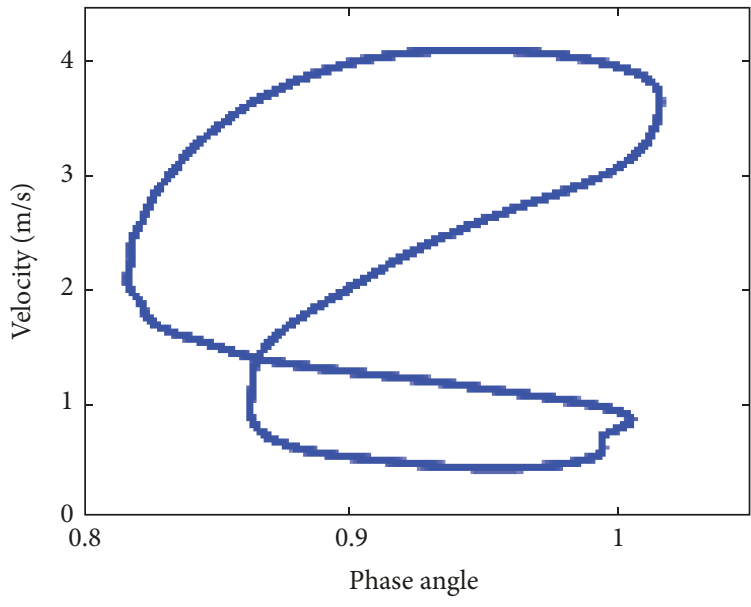

(b)

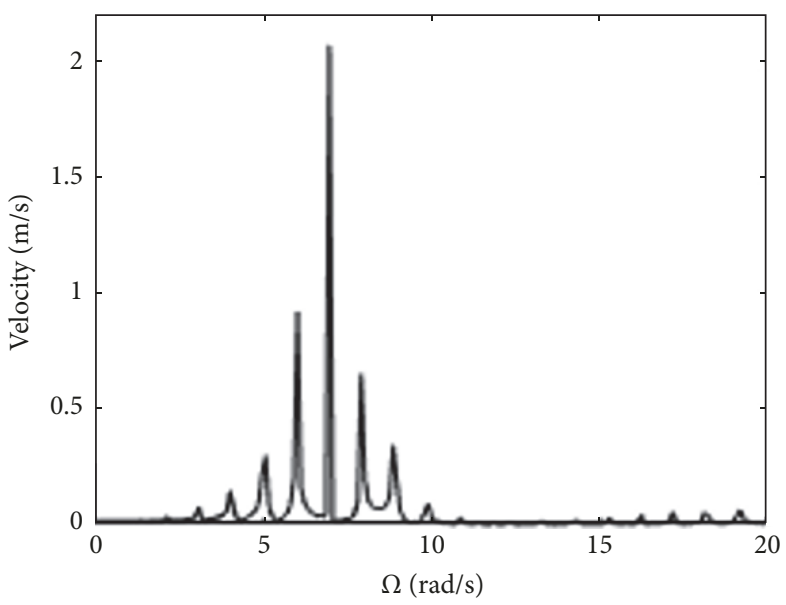

(c)

FIGURE 11: Quasiperiodic orbit at $\Omega=8.0 \mathrm{rad} / \mathrm{s}$ : (a) the phase portrait, (b) the Poincaré map, and (c) the frequency spectrum.

$\Omega=8.0,8.05$, or 8.15 , the system exhibits a quasiperiodic motion and the map forms a continuous closed orbit in the Poincaré section, as shown in Figures 11-13. As the forcing frequency $(\Omega)$ continues decreasing into region $\mathbf{A}$ (Figure 14), chaotic motion produces chatter vibration. The transition sequence for the system in this paper is from periodic motion to quasiperiodic motion and finally to chaotic motion. The particular features of these two descriptors characterize the chaotic behavior. The Poincaré map contains an infinite set of points, which are referred to as a strange attractor. The frequency spectrum of the chaotic motion is a continuous broad spectrum. The strange attractor and continuous Fourier spectrum are strong indicators of chaos. Figure 14 shows clear indications of chaotic behavior in region $\mathbf{A}$. Figures 14(a)-14(c), respectively, show the phase portraits, the Poincaré maps, and frequency spectra.

\section{Analysis of Chaotic Phenomena: Lyapunov Exponent and Lyapunov Dimension}

The analysis presented in Section 4 is insufficient to confidently identify chaotic motion in a suspension system. In this section, we adopt Lyapunov exponents to verify the existence of chaotic motion. In every dynamic system, the spectrum of Lyapunov exponents $(\lambda)$ [20] indicates variations in the lengths, areas, and volumes of the phase space. Verifying the existence of chaos requires calculation of only the largest exponent to indicate whether nearby trajectories diverge $(\lambda>0)$ or converge $(\lambda<0)$, on average. Any bounded motion within a system that has at least one positive Lyapunov exponent is defined as chaotic. The Lyapunov exponents for periodic motion are not positive.

Figure 15 plots the evolution of the largest Lyapunov exponent in a suspension system. This value is computed using the algorithm presented by Wolf et al. [20]. Figure 14 shows that chaotic motion began at approximately $\Omega=$ $7.965 \mathrm{rad} / \mathrm{s}$, because at point $\left(P_{1}\right)$, the sign of the largest Lyapunov exponent changed from negative to positive as the parameter, $\Omega$, slowly decreased. Between points $P_{1}$ and $P_{2}$, the largest Lyapunov exponent approached zero. The existence of a forcing frequency, $\Omega$, in these regions denotes the motion in the system as quasiperiodic. When the value of $\Omega$ is greater than $P_{1}$ (such as when $\Omega=8.2 \mathrm{rad} / \mathrm{s}$ ), the Lyapunov exponents obtained using (3) are $\lambda_{1}=-0.0010375$ and $\lambda_{2}=$ -0.0013232 . The sum of these values is $\lambda_{1}+\lambda_{2}=-0.0023607$; this negative value demonstrates that the motion of mass at these values eventually converges to a stable limit cycle. Using the Lyapunov exponents for a dynamical system where $\lambda_{1} \geq \cdots \geq \lambda_{n}$, Kaplan and Yorke [32] provided an estimation of the Lyapunov dimension, $d_{L}$, as follows:

$$
d_{L}=j+\frac{1}{\left|\lambda_{j+1}\right|} \sum_{i=1}^{j} \lambda_{i},
$$

where $j$ is the largest integer that satisfies $\sum_{i=1}^{j} \lambda_{i}>0$. This technique yields a Lyapunov dimension of $d_{L}=1$ for 


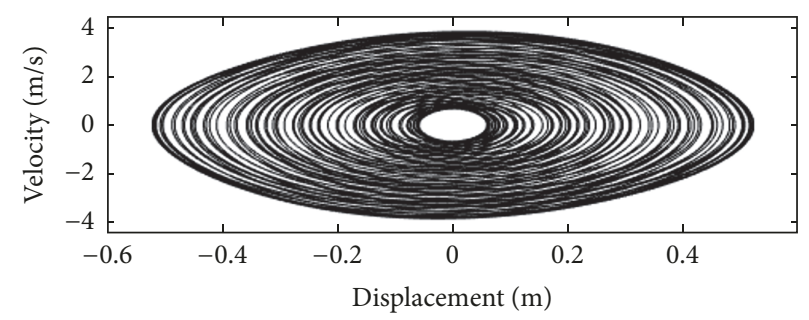

(a)

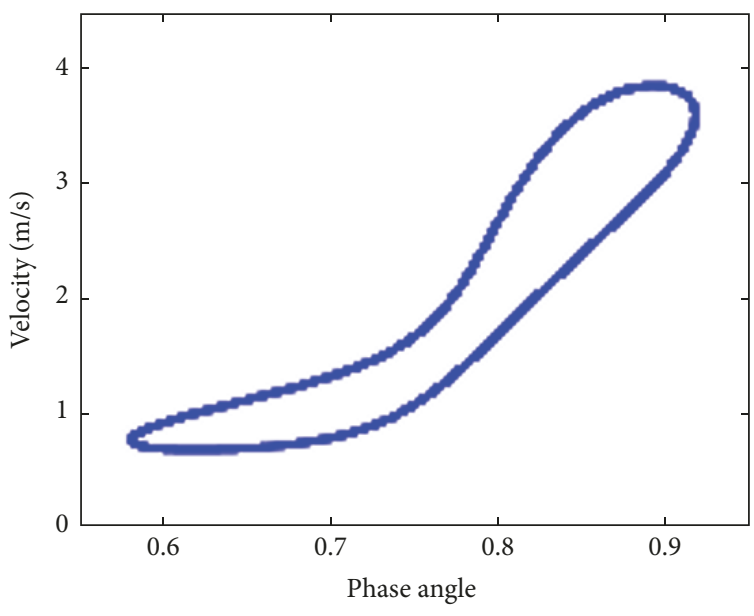

(b)

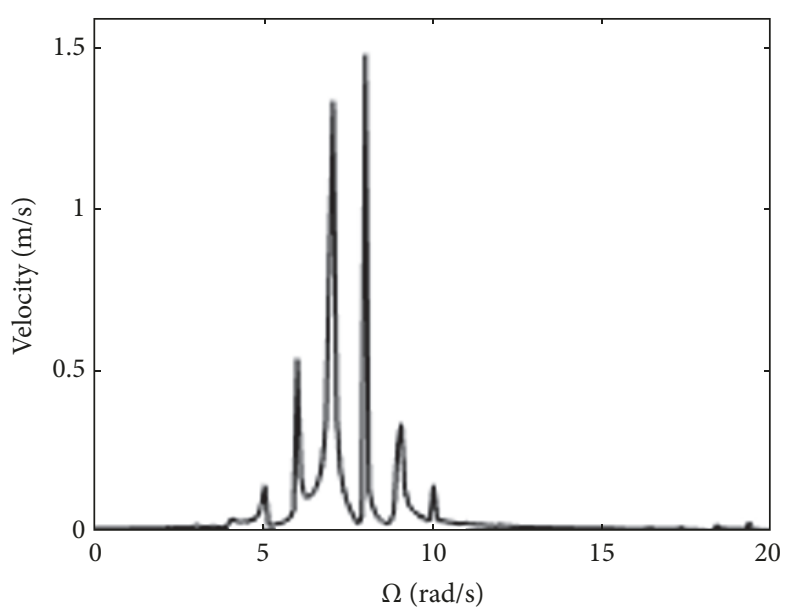

(c)

FIgURE 12: Quasiperiodic orbit at $\Omega=8.05 \mathrm{rad} / \mathrm{s}$ : (a) the phase portrait, (b) the Poincaré map, and (c) the frequency spectrum.

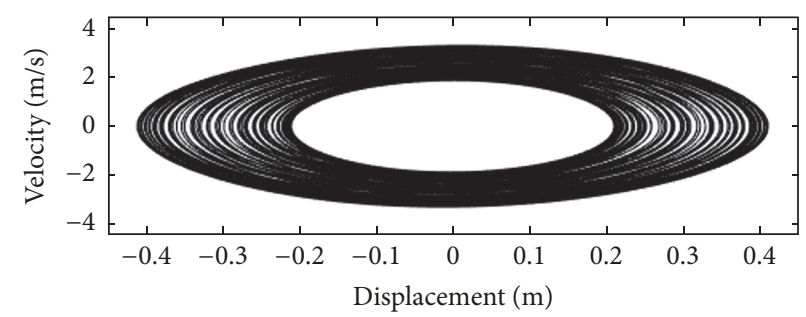

(a)

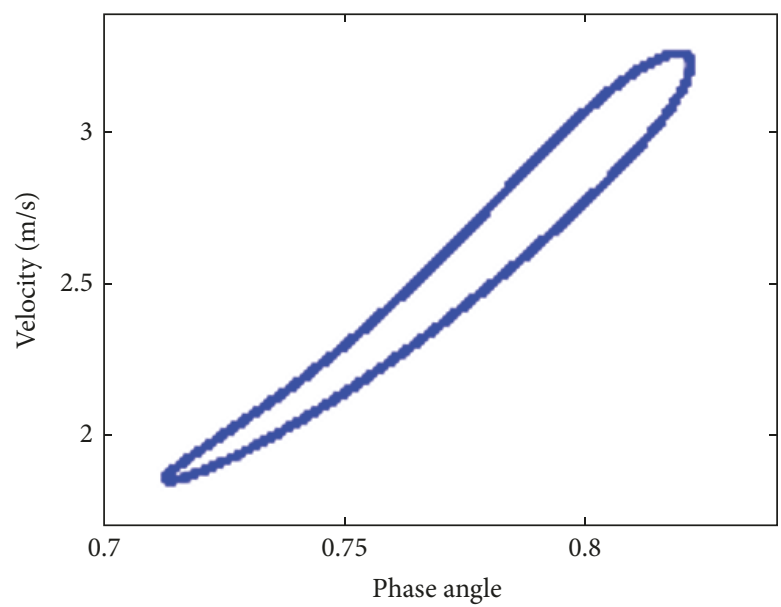

(b)

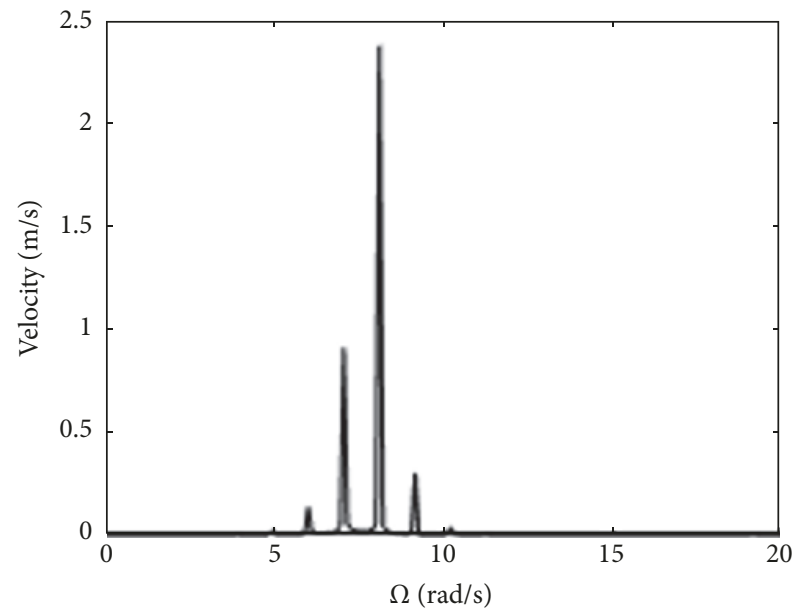

(c)

FIGURE 13: Quasiperiodic orbit at $\Omega=8.15 \mathrm{rad} / \mathrm{s}$ : (a) the phase portrait, (b) the Poincaré map, and (c) frequency spectrum. 


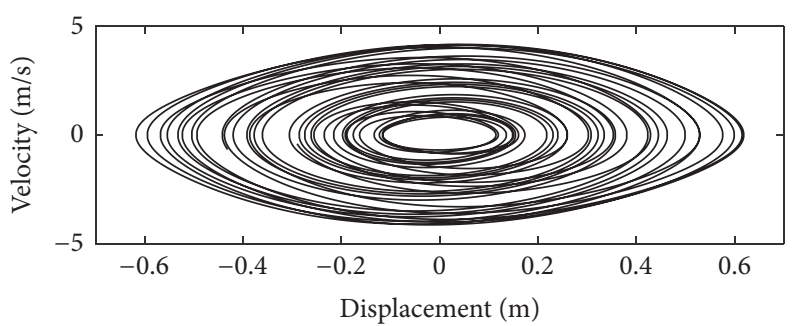

(a)

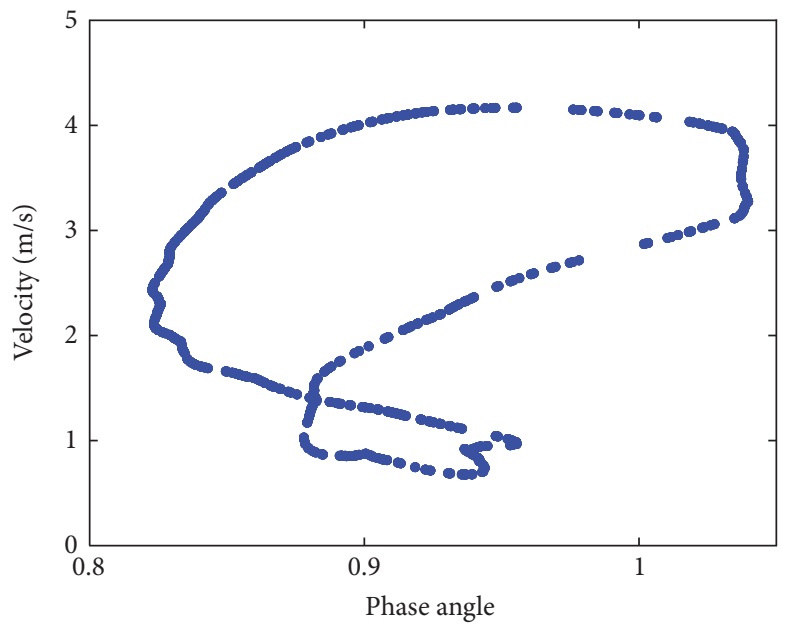

(b)

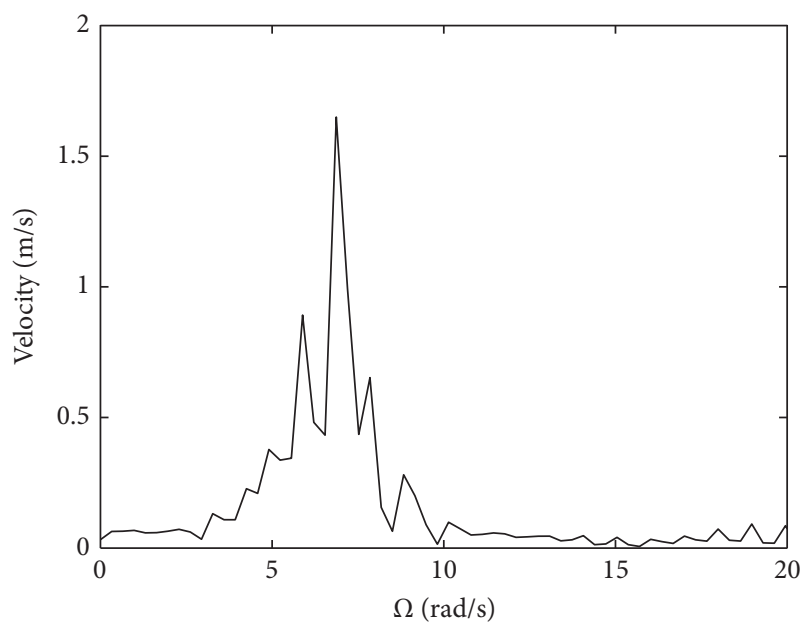

(c)

FIGURE 14: Chaotic motion at $\Omega=7.964 \mathrm{rad} / \mathrm{s}$ : (a) the phase portrait, (b) Poincaré map, and (c) the frequency spectrum.

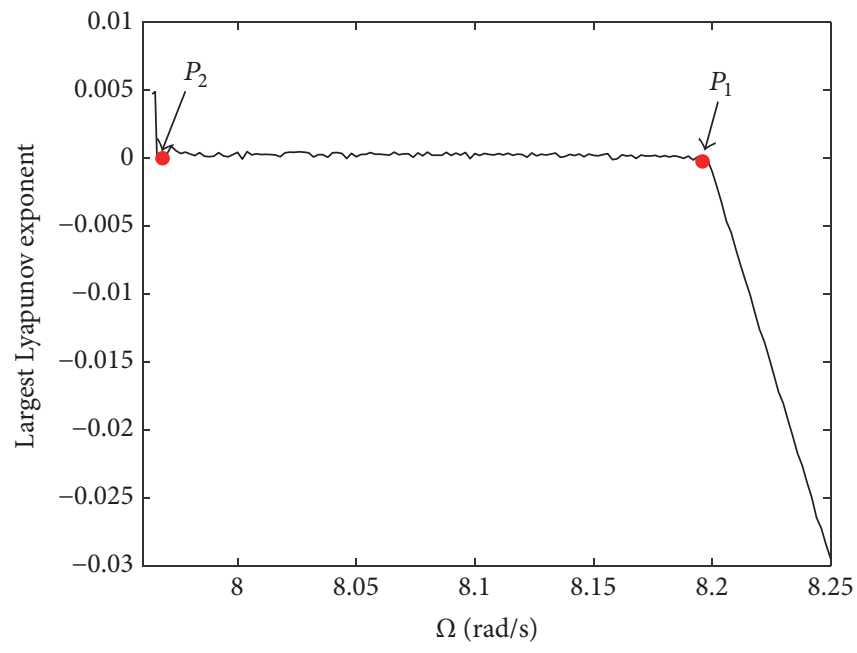

FIGURE 15: Evolution of the largest Lyapunov exponent.

$\Omega=8.2 \mathrm{rad} / \mathrm{s}$. The fact that the Lyapunov dimension is an integer means that the system has periodic motion. When parameter $\Omega$ increases beyond the bifurcation point (such as when $\Omega=7.964 \mathrm{rad} / \mathrm{s}$ ), the Lyapunov exponents are $\lambda_{1}=$ 0.0054396 and $\lambda_{2}=-0.092153$, and the Lyapunov dimension is $d_{L}=1.059$. In this case, the Lyapunov dimension is not an integer, indicating that the system exhibits chaotic motion.

\section{Controlling Chaos}

Improving the performance of a dynamic system and/or avoiding chaotic behavior require periodic motion, which is more important when working under specific conditions. Cai et al. [26] suggested a simple, effective control method that converts chaotic motions into periodic motion using the linear state feedback as a system variable. This approach 


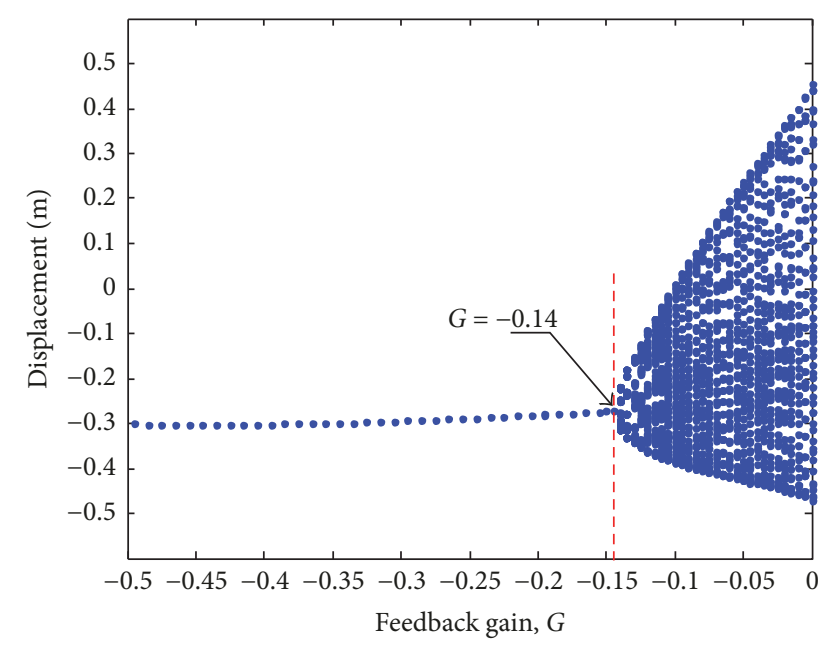

FIGURE 16: The bifurcation diagram for a system with state feedback control, where $G$ denotes the feedback gain.

to achieving an $n$-dimensional dynamic system is explained briefly as follows:

$$
\dot{x}=f(x, t),
$$

where $x(t) \in R^{n}$ is the state vector and $f=$ $\left(f_{1}, \ldots, f_{i}, \ldots, f_{n}\right)$, where $f_{i}$ is a linear or a nonlinear function and $f$ includes at least one nonlinear function. If $f_{k}(x, t)$ is a nonlinear function that gives chaotic motion in (20), then only one term of the state feedback for the available system variable, $x_{m}$, is added to the equation that includes $f_{k}$, as follows:

$$
\dot{x}_{k}=f_{k}(x, t)+G x_{m}, \quad k, m \in\{1,2, \ldots, n\},
$$

where $G$ is the feedback gain. Other functions remain in their original forms.

System (3) with state feedback control can be written as follows:

$$
\begin{aligned}
\frac{d y_{1}}{d t}= & y_{2} \\
\frac{d y_{2}}{d t}= & -\omega^{2} y_{1}-B_{1} y_{1}^{3}-B_{2} y_{2}-B_{3} y_{2}^{3} \\
& +\widetilde{a} \Omega^{2} \sin (\Omega t)+G y_{2} .
\end{aligned}
$$

In the absence of state feedback control, $G=0$ and (3) describes chaotic motion when $\Omega=7.964 \mathrm{rad} / \mathrm{s}$. Figure 16 shows the resulting bifurcation diagram for a system with state feedback control. This figure clearly shows that chaotic motion occurs at approximately $G>-0.14$ and disappears at approximately $G \leq-0.14$. When feedback gain, $G$, falls below -0.14 , stable periodic motion occurs. As shown in Figure 17, a control signal is applied to the system at $35 \mathrm{~s}$ to govern the chaotic oscillation. In this manner, state feedback control can be used to suppress chaotic motion and improve the performance of a vehicle's suspension system. The form

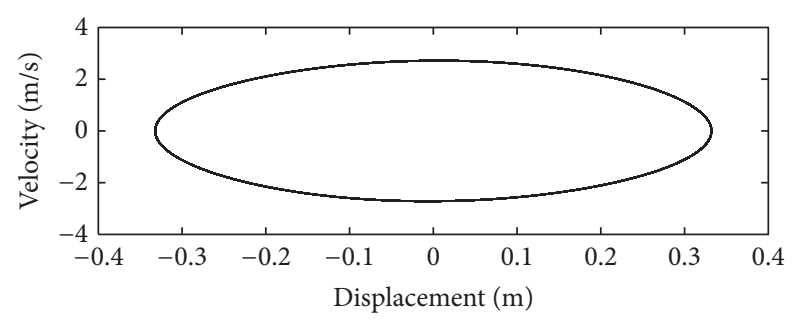

(a)

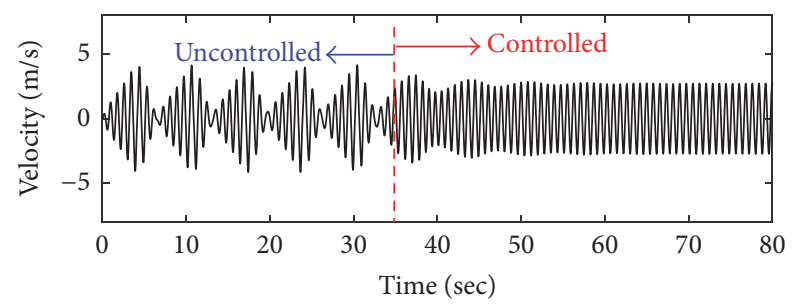

(b)

FIgure 17: Transforming chaotic motion to a desired period-one orbit for $G=-0.3$ and $\Omega=7.964 \mathrm{rad} / \mathrm{s}$ : (a) the phase portrait for the controlled system when the state feedback control signal is introduced after $35 \mathrm{~s}$ and (b) the time responses of the velocity.

of the control gain in the original system equation (1) is as follows:

$$
\widetilde{G}=G \cdot m \text {. }
$$

The physical meaning of gain, $\widetilde{G}$, is control gain and the unit of the gain is $\mathrm{N} \cdot \mathrm{s} / \mathrm{kg} \cdot \mathrm{m}$.

In many general systems that involve chaotic dynamics, this state feedback technique does not require preliminary or on-line analysis of the system dynamics and can be implemented by adding the feedback of a suitable variable to the original system with sufficient control gain to suppress the development of chaos. In this regard, the state feedback control technique is simple and effective approach to chaos suppression.

\section{Conclusions}

This study examined the nonlinear dynamic behavior in a vehicle suspension system with hysteretic damping. Our objective was to develop a method to control chaotic vibration. Dynamic behavior was observed over the entire range of parameter values of the bifurcation sets and bifurcation diagram. Using bifurcation analysis, we identified a codimension-two bifurcation structure with double-zero degeneracy in the system. The bifurcation diagram indicates the existence of quasiperiodic orbit and chaotic motion, thereby demonstrating the effectiveness of using Lyapunov exponents as a tool for identifying chaotic motion. Finally, we proposed a state feedback control method to suppress chaotic motions and improve the ride provided by an automotive suspension system. Simulation results confirm the feasibility of the proposed method. Our results provide insight into the operating conditions under which undesirable dynamic motion takes place in vehicle suspension systems. Our results 
also provide a useful reference for engineers involved in the design of control systems for vehicle suspensions. It should be noted that in practical engineering, phenomena such as actuator faults, time-varying delays, unmodeled dynamics, and dynamics disturbances often occur simultaneously, and these problems are beyond the scope of the current study. In future work, we will seek to control nonlinear systems with time-varying delays.

\section{Conflicts of Interest}

The authors declare that there are no conflicts of interest regarding the publication of this paper.

\section{Acknowledgments}

The authors would like to thank the Ministry of Science and Technology of the Republic of China, Taiwan, for financially supporting this research under Contract no. MOST 105-2221E-212-016.

\section{References}

[1] H. Chen, Z.-Y. Liu, and P.-Y. Sun, "Application of constrained script $H_{\infty}$ control to active suspension systems on half-car models," Journal of Dynamic Systems, Measurement, and Control, vol. 127, no. 3, pp. 345-354, 2005.

[2] X. Song, M. Ahmadian, S. Southward, and L. Miller, "Parametric study of nonlinear adaptive control algorithm with magnetorheological suspension systems," Communications in Nonlinear Science and Numerical Simulation, vol. 12, no. 4, pp. 584-607, 2007.

[3] L.-M. Gui, W.-K. Shi, and W. Liu, "A semi-active suspension design for off-road vehicle base on Magneto-rheological technology," in Proceedings of the 2012 9th International Conference on Fuzzy Systems and Knowledge Discovery, FSKD 2012, pp. 2565-2568, China, May 2012.

[4] F. Tu, Q. Yang, C. He, and L. Wang, "Experimental Study and Design on Automobile Suspension Made of Magneto-Rheological Damper," in Proceedings of the International Conference on Future Energy, Environment, and Materials, FEEM 2012, pp. 417425, Hong Kong, April 2012.

[5] P. W. Nugroho, H. Du, W. Li, and G. Alici, "Implementation of Adaptive Neuro Fuzzy Inference System controller on magneto rheological damper suspension," in Proceedings of the 2013 IEEE/ASME International Conference on Advanced Intelligent Mechatronics: Mechatronics for Human Wellbeing, AIM 2013, pp. 1399-1403, Australia, July 2013.

[6] Arnold VI. Geometric Methods in the Theory of Ordinary Differential Equations. New York: Springer-Verlag. 1988.

[7] P. J. Holmes, "Bifurcations to divergence and flutter in flowinduced oscillations: a finite dimensional analysis," Journal of Sound and Vibration, vol. 53, no. 4, pp. 471-503, 1977.

[8] J. Guckenheimer and P. J. Holmes, Nonlinear Oscillations, Dynamical System and Bifurcation of Vector Fields, SpringerVerlag, New York, 2013.

[9] C.-Y. Tseng and P.-C. Tung, "Dynamics of nonlinear structure with magnetic actuator," Japanese Journal of Applied Physics, vol. 34, no. 1, pp. 374-382, 1995.
[10] K. Yagasaki, "Codimension-two bifurcations in a pendulum with feedback control," International Journal of Non-Linear Mechanics, vol. 34, no. 6, pp. 983-1002, 1999.

[11] R. Tian, Q. Cao, and S. Yang, "The codimension-two bifurcation for the recent proposed SD oscillator," Nonlinear Dynamics, vol. 59, no. 1-2, pp. 19-27, 2010.

[12] S. Yin, P. Shi, and H. Yang, "Adaptive Fuzzy Control of StrictFeedback Nonlinear Time-Delay Systems With Unmodeled Dynamics," IEEE Transactions on Cybernetics, vol. 46, no. 8, pp. 1926-1938, 2016.

[13] S. Yin, H. Yang, H. Gao, J. Qiu, and O. Kaynak, "An adaptive NN-based approach for fault-tolerant control of nonlinear time-varying delay systems with unmodeled dynamics," IEEE Transactions on Neural Networks and Learning Systems, vol. 28, no. 8, pp. 1902-1913, 2017.

[14] S. Li, S. Yang, and W. Guo, "Investigation on chaotic motion in hysteretic non-linear suspension system with multi-frequency excitations," Mechanics Research Communications, vol. 31, no. 2, pp. 229-236, 2004.

[15] R. D. Naik and P. M. Singru, "Resonance, stability and chaotic vibration of a quarter-car vehicle model with time-delay feedback," Communications in Nonlinear Science and Numerical Simulation, vol. 16, no. 8, pp. 3397-3410, 2011.

[16] M. Borowiec, G. Litak, and M. I. Friswell, "Nonlinear response of an oscillator with a magneto-rheological damper subjected to external forcing," Applied Mechanics and Materials, vol. 5-6, pp. 277-284, 2006.

[17] G. Litak, M. Borowiec, M. I. Friswell, and K. Szabelski, "Chaotic vibration of a quarter-car model excited by the road surface profile," Communications in Nonlinear Science and Numerical Simulation, vol. 13, no. 7, pp. 1373-1383, 2008.

[18] J. Marzbanrad and A. Keshavarzi, "Chaotic vibrations of a nonlinear air suspension system under consecutive half sine speed bump," Indian Journal of Science and Technology, vol. 8, pp. 72-84, 2015.

[19] J. Marzbanrad and A. Keshavarzi, "A Chaotic viewpoint on a quarter-car air suspension model with numerical and experimental analysis[J]," Journal of Cahiers Des Sciences, vol. 23, 2014.

[20] A. Wolf, J. B. Swift, H. L. Swinney, and J. A. Vastano, "Determining Lyapunov exponents from a time series," Physica D: Nonlinear Phenomena, vol. 16, no. 3, pp. 285-317, 1985.

[21] E. Ott, C. Grebogi, and J. A. Yorke, “Controlling chaos," Physical Review Letters, vol. 64, no. 11, pp. 1196-1199, 1990.

[22] E. R. Hunt, "Stabilizing high-period orbits in a chaotic system: the diode resonator," Physical Review Letters, vol. 67, no. 15, pp. 1953-1955, 1991.

[23] C. Cai, Z. Xu, W. Xu, and B. Feng, "Notch filter feedback control in a class of chaotic systems," Automatica, vol. 38, no. 4, pp. 695701, 2002.

[24] G. Litak, M. Borowiec, M. Ali, L. M. Saha, and M. I. Friswell, "Pulsive feedback control of a quarter car model forced by a road profile," Chaos, Solitons \& Fractals, vol. 33, no. 5, pp. 16721676, 2007.

[25] H. Du, K. Y. Sze, and J. Lam, "Semi-active $H_{\infty}$ control of vehicle suspension with magneto-rheological dampers," Journal of Sound and Vibration, vol. 283, no. 3-5, pp. 981-996, 2005.

[26] C. Cai, Z. Xu, and W. Xu, "Converting chaos into periodic motion by state feedback control," Automatica, vol. 38, no. 11, pp. 1927-1933, 2002.

[27] M. Ashkenazi and S.-N. Chow, "Normal forms near critical points for differential equations and maps.," IEEE Transactions 
on Circuits and Systems II: Express Briefs, vol. 35, no. 7, pp. 850862, 1988.

[28] Y. N. Bibikov, Local theory of nonlinear analytic ordinary differential equations, vol. 702 of Lecture Notes in Mathematics, Springer-Verlag, Berlin, 1979.

[29] L. O. Chu and H. Kokubu, "Normal Forms for Nonlinear Vector Fields- Part I: Theory and Algorithm," IEEE Transactions on Circuits and Systems II: Express Briefs, vol. 35, no. 7, pp. 863-880, 1988.

[30] P. Holmes, "dynamics of a nonlinear oscillator with feedback control. i: local analysis," Journal of Dynamic Systems, Measurement, and Control, vol. 107, no. 2, pp. 159-165, 1985.

[31] IMSL, Inc., Users Manual IMSL MATH/LIBRARY, 1989.

[32] J. L. Kaplan and J. A. Yorke, "Chaotic behavior of multidimensional difference equations," in Functional Differential Equations and Approximation of Fixed Points (Proc. Summer School and Conf., Univ. Bonn, Bonn, 1978), vol. 730 of Lecture Notes in Math., pp. 204-227, Springer, Berlin, Germany, 1979. 


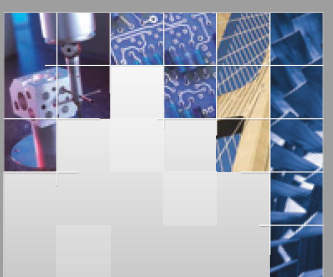

\section{Enfincering}
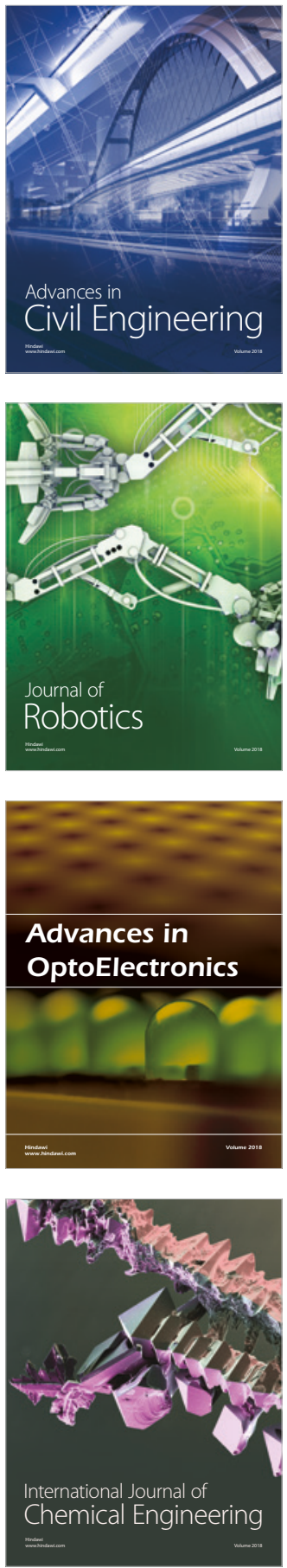

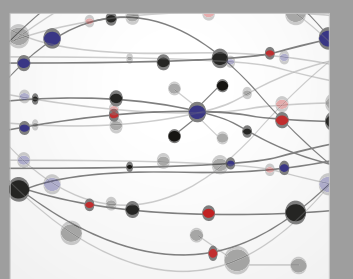

\section{Rotating \\ Machinery}

The Scientific World Journal

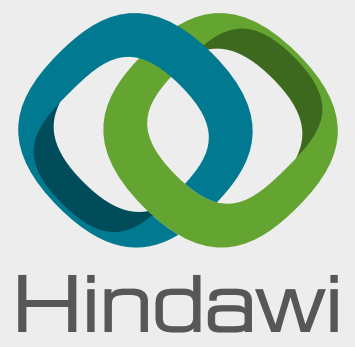

Submit your manuscripts at

www.hindawi.com
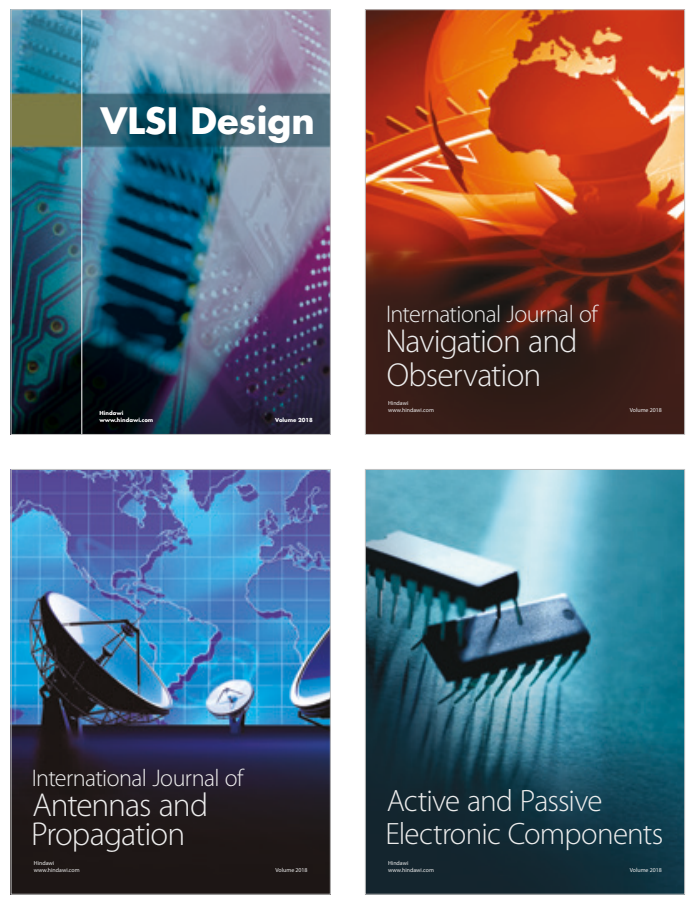
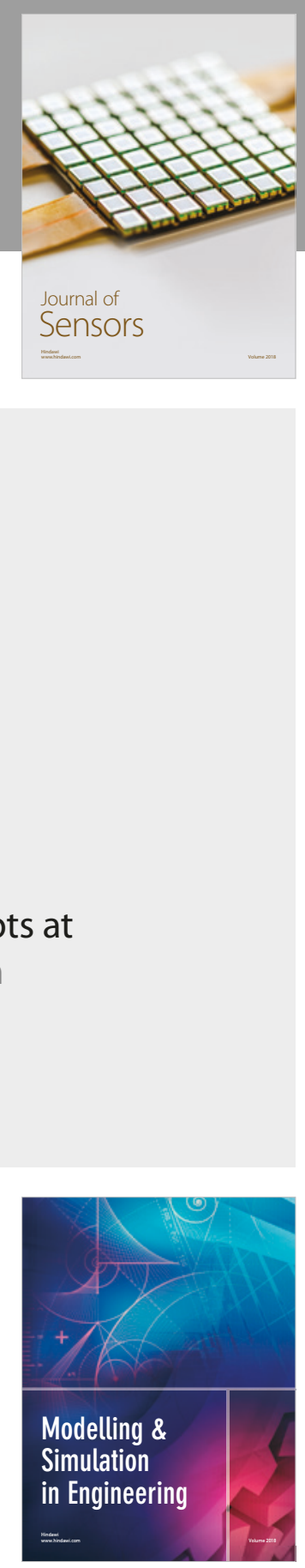

\section{Advances \\ Multimedia}
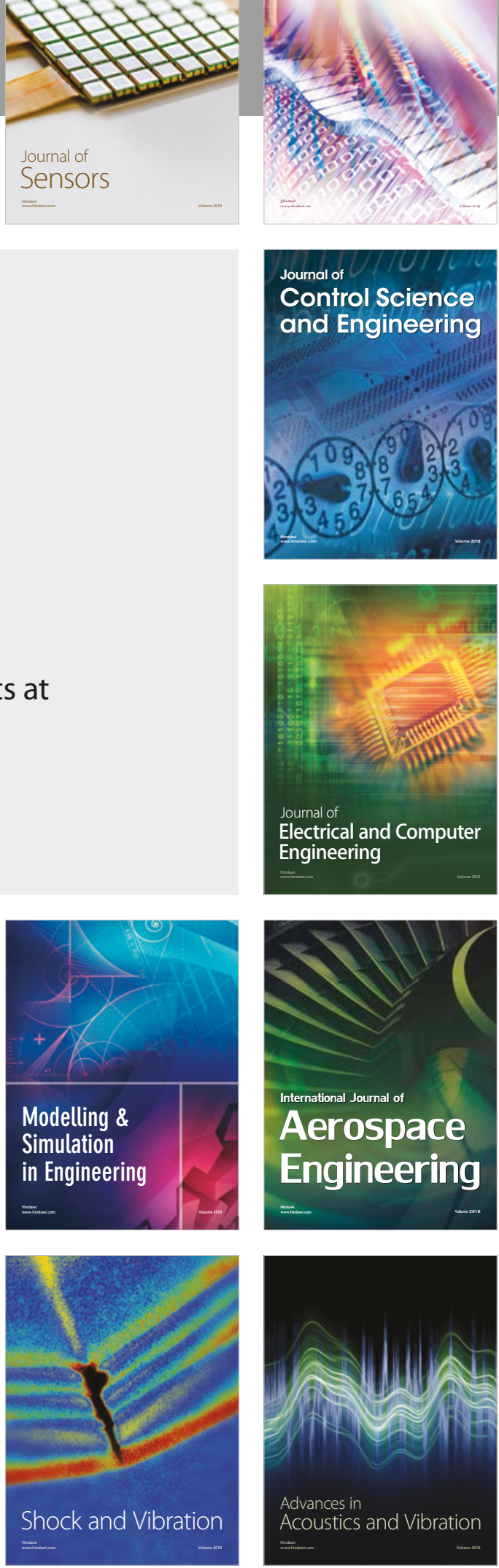